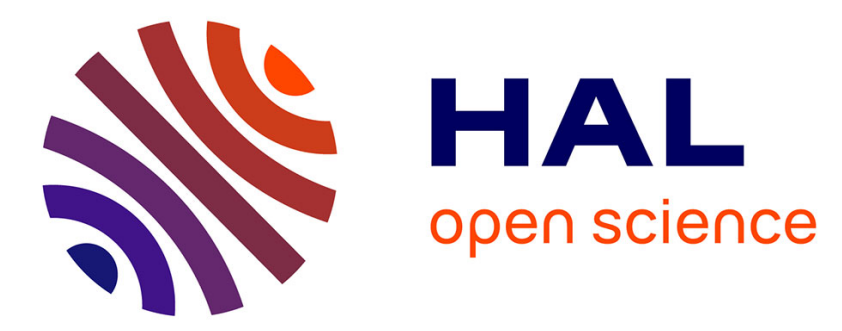

\title{
BEM Reference Signal Estimation for an Airborne Passive Radar Antenna Array
}

Clément Berthillot, Agnès Santori, Olivier Rabaste, Dominique Poullin, Marc Lesturgie

\section{- To cite this version:}

Clément Berthillot, Agnès Santori, Olivier Rabaste, Dominique Poullin, Marc Lesturgie. BEM Reference Signal Estimation for an Airborne Passive Radar Antenna Array. IEEE Transactions on Aerospace and Electronic Systems, 2017, 53 (6), pp.2833 - 2845. 10.1109/TAES.2017.2716458 . hal01705163

\section{HAL Id: hal-01705163 \\ https://hal.science/hal-01705163}

Submitted on 9 Feb 2018

HAL is a multi-disciplinary open access archive for the deposit and dissemination of scientific research documents, whether they are published or not. The documents may come from teaching and research institutions in France or abroad, or from public or private research centers.
L'archive ouverte pluridisciplinaire HAL, est destinée au dépôt et à la diffusion de documents scientifiques de niveau recherche, publiés ou non, émanant des établissements d'enseignement et de recherche français ou étrangers, des laboratoires publics ou privés. 


\title{
BEM Reference Signal Estimation for an
}

\section{Airborne Passive Radar Antenna Array}

\author{
Clément Berthillot* $\dagger$, Agnès Santori ${ }^{\dagger}$, Olivier Rabaste $\ddagger$, Dominique Poullin ${ }^{\ddagger}$ and Marc Lesturgie* $\ddagger$ \\ *SONDRA - Plateau du Moulon - 91192 Gif/Yvette, France \\ ${ }^{\dagger}$ CReA - French Air Force Research Center \\ BA701 - 13661 Salon-de-Pce, France - Email: clement.berthillot@intradef.gouv.fr \\ ‡ONERA (The French Aerospace Lab) - BP 80100 - 91123 Palaiseau Cedex, France
}

\begin{abstract}
In this article, we consider an airborne passive radar using DVB-T OFDM signals. In such an application, the channel composite nature and the mobility of the receiver deeply alter the signal and therefore degrade the decoding processing. Classic methods experience difficulties in dealing with such channel impacts. In order to reconstruct a proper reference signal, we propose here a channel estimation method that exploits both antenna diversity and a Basis Expansion Model (BEM) modeling the channel time-variations. The application of this method to experimental in-flight recorded data demonstrates the performance improvement provided by the proposed method over the classic demodulation and other methods developed to cope with InterCarrier Interferences (ICI).
\end{abstract}

Index Terms

Passive radar, Airborne Radar, Signal Processing, Channel Estimation, OFDM, DVB-T, Basis Expansion Model, Antenna Diversity

\section{INTRODUCTION}

Passive radars have attracted a lot of attention and concern during the last decade. Signal processing methods have been developed for all available transmitters of opportunity such as FM [1], Digital Audio Broadcasting (DAB) [2], Digital Video Broadcasting - Terrestrial (DVB-T) [3], [4], [5], and even Global System for Mobile (GSM) or Wifi [6]. This topic has nowadays reached a certain maturity. Systems have been designed and tested for different applications : at first air surveillance [7], but also Ground Moving Target Identification (GMTI) and Sea Surveillance [8], [9]. Besides passive radar characteristics, such as low weight, low power consumption and stealthyness, they are good candidates to airborne applications. As a consequence, analyses have been recently drawn so as to study the applicability of passive airborne radars. Reference [10] describes the overall concept, whereas [11] provides an insight of the necessary system performance, and [12] simulates the received signal and analyses the ambiguity function. The first experiments have allowed to examine ground clutter [13], and to consider the detection capabilities of such a system [14], [15], [16] based on FM signal. Moreover [17], [18] show the benefits of a suitable bistatic geometry to face ground clutter echoes for DVB-T SAR imagery. 
In this context, the SONDRA laboratory, the Research Center of the French Air force (CReA), and the French Aerospace Lab (ONERA) have developed a complete passive receiver, including a specific antenna array. The system flew on the ONERA motorglider, named BUSARD, so as to detect airborne targets using the OFDM modulated DVB-T signals [19].

In addition the detection step needs a relevant reference signal. This reference signal can be obtained either using a specific dedicated channel pointed towards the transmitter direction, or estimated from the received signal, possibly by exploiting the specific properties of the transmitted signal. The latter is especially true in case of digital modulations, DAB and DVB for instance. The signal is demodulated and decoded in order to remove any multipath effect, before it is re-encoded and modulated again to create the proper multipath-free reference signal. This procedure presents the huge advantage, to suppress most of the multipath effect and target echoes. However it can not reproduce the transmitter and receiver behaviour (filter, noise, etc.).

All previously cited articles take for granted that a relevant reference signal is available. However the moving aeronautical environment introduces heavy constraints. Aeronautical channel models reasonably assume that the transmitter and the receiver are Line-Of-Sight (LOS). So, to describe the en-route scenario, [20] proposes to divide the aeronautical channel into two contributions: a LOS, and a more stochastic diffuse backscattered component. A ground reflection component may be associated to this two-ray model [21]. This is all the more relevant that, in the current case, transmitters are generally tilted to the ground. This multipath environment implies that the aeronautical channel is strongly composite.

Moreover the aeronautical environment involves large distances and as a consequence potentially large multipath delays compared to the symbol period. Stronger ground echoes may for instance present a delay corresponding to the propagation time necessary to travel the distance from transmitter to ground and then from ground to receiver, that is about twice the receiver's altitude. Thus the channel coherence bandwidth may be far less than the almost $8 \mathrm{MHz}$ bandwidth of DVB-T signal. The aeronautical channel is hence frequency selective, which implies that deep fades may corrupt the Channel Frequency Response (CFR).

In addition it is also characterised by the mobility of the receiver. As a first consequence, the Channel Impulse Response (CIR) may fluctuate over time. Each component of the multipath aeronautical channel may show a different Doppler frequency. This differential Doppler shift degrades the orthogonality among the subcarriers and may introduce ICI. In other words, the received power does not only come from the desired subcarrier but also from its neighboors. The elementary symbol to be read is therefore corrupted by these interferences.

Usually, the OFDM channel estimation uses pilots, that is to say, symbols that are known at the receiver, to get the Least Squares (LS) estimate of the CFR at pilot subcarrier indexes. The whole CFR is then inferred thanks to frequency domain interpolation. But it does not take into account time variations or potential ICI. Therefore, the literature proposes a large solution panel to estimate a time-varying channel in case of OFDM signals. First, some articles propose to expand interpolation to the time and frequency domains [22], [23]. As the estimation methods need to catch the channel fluctuations, [24] derives a linear model based either on consecutive symbols, or only one symbol including its guard interval. And [25] expands it to higher order polynomial.

Besides methods were derived so as to benefit from all possible channel information. Especially [23] proposes a way to evaluate the precise channel length. Reference [26] considers a solution based on the combination of a 
Minimum Description Length (MDL) method that provides the number of active taps, and the ESPRIT algorithm that estimates their respective delays as detailed in [27]. It participates to decrease the number of coefficients to estimate [28].

When ICI is preponderent, it must be estimated but also cancelled. Several iterative solutions have been presented. Reference [29] proposes to decode and then re-encode the data to make use of virtual pilots, whereas [30] derives a QR-factorization based equalizer to remove ICI.

To represent time varying channel, Basis Expansion Model (BEM) have also been introduced [31], [32]. The general time domain model has then been adjusted to OFDM signals [33], [34], [35]. The BEM estimation principle consists in decomposing the CIR on an orthonormal basis of time-varying functions restricted over a certain span, to model the taps time variations. It includes Polynomial BEM (P-BEM). However many BEM types exist such as the Complex-Exponential BEM (CE-BEM), the Generalized CE-BEM (GCE-BEM), the Karhunen-Loeve BEM (KL-BEM) [36] or the Prolate Spheroidal BEM (PS-BEM) [37]. The BEM type may be chosen so as to fit a possible statistical channel a priori. Thanks to the BEM, the number of coefficients to estimate deeply reduces.

Reference [38] points the benefits of using the BEM on several consecutive OFDM symbols. In fact, the article shows that this solution implies to increase the basis size so as to model the intra-symbol channel variations along this extended time window. A larger basis means more unknown parameters to estimate. Indeed the CIR needs to be evaluated for each signal sample. Yet the number of CFR samples, i.e. the number of pilots, remains limited. Thus only a few symbols may be taken into account.

These solutions focus on rapidly time varying channel, that are mainly characterised by the ICI impact. Yet the channel faced during our airborne passive radar experimentation, appears to be slow-time varying, and shows a wide diffuse backscattered component. Simple study on real data proves indeed that most of classic intra-symbol BEM coefficients are negligible. So we developed an original solution that is able to catch the composite nature of the channel and its inter-symbol variations instead of intra-symbol fluctuations. Consequently we derive in this article a CE-BEM estimator tuned to OFDM signal based on a large block of consecutive symbols. Contrary to intra-symbol channel estimation that demands to estimate the CIR at every sample time, our method needs to evaluate the CIR only once per symbol. Thus the complexity is severely decreased. However this method needs some adjustment, as it appears that taking into account several consecutive symbols generates a certain phase rotation of the constellation due to the shift of the pilot position from one symbol to the next one. We describe and explain this phenomenon in this article and propose solutions that correct this phase rotation. The proposed method has been implemented and tested on real data recorded during a flight test that happened in october 2015. The results confirm its ability to improve the channel estimation, and consequently the reference signal reconstruction.

Section II introduces the signal model and the environment constraints. Section III details the proposed method. Finally section IV presents the experimental results.

\section{SignAL MODEL}

\section{A. DVB-T Signal}

The DVB-T signal is based on an OFDM modulation. [19]. Table I summarizes the 8K-mode parameters. 
TABLE I

DVB-T MODE 8K SIGNAL CONFIGURATION

\begin{tabular}{|c||c|}
\hline \multicolumn{1}{|c|}{ Constellation } & 64-QAM \\
\hline Subcarrier number, $N$ & 8192 \\
\hline Useful Subcarrier number & 6817 \\
\hline Pilot number, $L$ & 568 \\
\hline Guard Interval Samples, $G$ & 1024 \\
\hline Symbol useful duration, $T_{u}$ & $896 \mu \mathrm{s}$ \\
\hline Subcarrier spacing, $1 / T_{u}$ & $1116 \mathrm{~Hz}$ \\
\hline Bandwidth & $7.61 \mathrm{MHz}$ \\
\hline
\end{tabular}

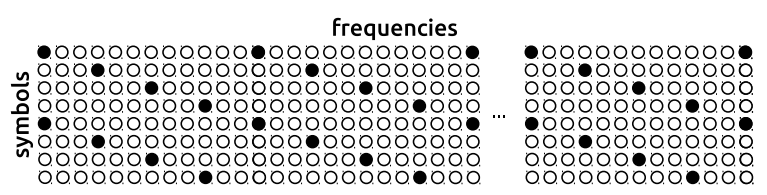

Fig. 1. DVB-T scattered pilot pattern - black dots represent the pilot location

According to OFDM principles, first binary data are mapped into elementary symbols of a complex constellation. $N$ elementary symbols constitute an OFDM symbol $\mathbf{X}$. These elementary symbols are placed on $N$ orthogonal subcarriers. $L$ pilots are inserted among these $N$ subcarriers. These pilots are encoded using a BPSK modulation and transmitted at boosted power. They are periodically distributed every $D_{f}=12$ subcarriers and shifted by 3 subcarriers from one OFDM symbol to the next. As a consequence, the location of these scattered pilots is periodic in every four OFDM symbols - Fig.1. Let $P_{n}(m)$ for $m=0 \ldots L-1$, denote their index for the $n^{\text {th }}$ OFDM symbol:

$$
P_{n}(m)=m D_{f}+d_{n}
$$

where $d_{n}=3 \bmod _{4}(n)$ and $\bmod$ denotes the modulo operator. $d_{n}$ represents the fact that pilot indexes are translated by 3 subcarriers from one OFDM symbol to the next. But, as it is based on the modulo operator, this structure is periodically repeated every 4 OFDM symbols as illustrated by the black dots in Fig.1. For instance $d_{0}=3 \times \bmod _{4}(0)=0, d_{1}=3 \times \bmod _{4}(1)=3$ and $d_{1}=d_{5}$.

The modulated signal is built this way in the frequency domain. The time domain equivalent symbol is inferred from $\mathbf{X}$ by applying an inverse discrete Fourier transform: $\mathbf{x}=\mathbf{F}^{H} \mathbf{X}$, where $\mathbf{F}$ is the unitary discrete Fourier matrix, and ${ }^{H}$ symbolizes the hermitian transpose. A guard interval is concatenated to the front of $\mathbf{x}$, which is composed of its last $G$ samples. Fig.2 represents the modulation of an OFDM symbol. In case of a stationary receiver, terrestrial for instance, the guard interval ensures the orthogonality among the subcarriers if the maximum delay does not exceed the length of this interval. However the aeronautical mobile environment undermines this property. Namely all the received paths coming from different direction, show different Doppler shifts. As a consequence the subcarriers are no longer orthogonal.

Let $T_{s}$ and $F_{s}$ denote respectively the sample period and rate. The pilots provide $L$ samples of the CFR. As a consequence, it gives access to the CIR over only $L T_{s}$ delays. More precisely, the DVB-T norm includes a guard band that prevents from interferences with neighbouring transmitted channel. The CFR $L$ samples are located in 


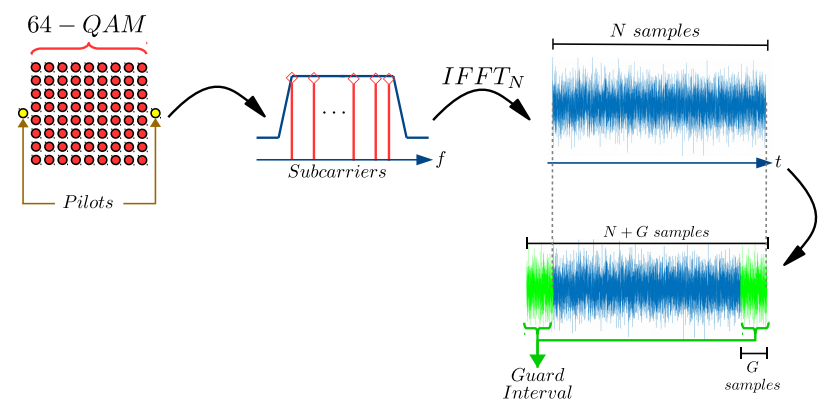

Fig. 2. OFDM signal modulation

the useful band $B w_{u}$ :

$$
B w_{u}=L D_{f} \frac{F_{s}}{N}
$$

It implies that the CIR may be observed at sample period:

$$
T_{s}^{(C I R)}=\frac{N}{L D_{f}} T_{s}
$$

Yet, as $D_{f} \neq N / L, T_{s}^{(C I R)} \neq T_{s}$. So the CIR sample period differs from the signal one. As a consequence virtual pilots have to be considered so as to cover the whole band. This equates to zero-padding. It is as if there were: $L^{\prime}=\left\lfloor\frac{N}{D_{f}}\right\rfloor$ pilots. Thus the CIR is sampled as so:

$$
T_{s}^{(C I R)^{\prime}}=\frac{N}{L^{\prime} D_{f}} T_{s} \simeq T_{s}
$$

It can be consequently inferred that the maximum channel delay that may be observed is $L^{\prime} T_{s}$, where $L=682$. That represents a maximum delay of $75 \mu \mathrm{s}$, or $22.4 \mathrm{~km}$. In the following we assume, this is the channel length. For sake of simplicity, $L$ will refer to $L^{\prime}$.

\section{B. The Aeronautical Channel}

As our interest is focused on low altitude targets, the receiver altitude is limited up to $z_{R X}=1500 \mathrm{~m}$. We assume that the transmitter is located at the same altitude - see 4(b): $z_{T X}=z_{R X}$. Therefore the main echoes are mostly concentrated during the first $10 \mu \mathrm{s}$, that is at most $100 \times T_{s}$. First it points the fact that the delay spread do not exceed the $1024 \times T_{s}$ guard interval. So we neglect the intersymbol interferences. Moreover it implies, the channel coherence bandwidth is about $100 \mathrm{kHz}$, far less than the almost $8 \mathrm{MHz}$. As previously stated, it confirms that the aeronautical channel is frequency selective.

The channel taps $h_{(n, l)}^{(t)}$ denote the complex path gain for the $n^{t h}$ symbol and path delay $l T_{s}$. The received signal for symbol $n$ can be written in a matrix way:

$$
\mathbf{y}_{n}=\mathbf{H}_{n}^{(t)} \mathbf{x}_{n}+\mathbf{u}_{n}
$$

where $\mathbf{u}_{n}$ is an additive white Gaussian noise, and where the $l^{\text {th }}$ diagonal of $\mathbf{H}_{n}^{(t)}$ corresponds to the $l^{t h}$ delay 


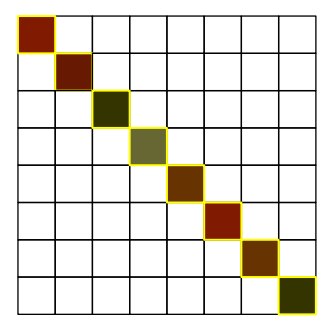

(a)

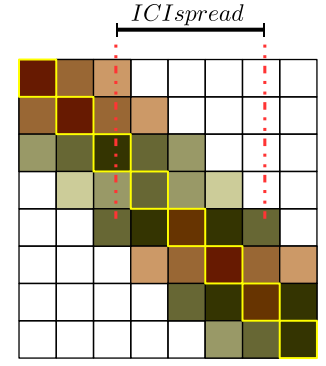

(b)

Fig. 3. Channel matrix without (a) and with (b) ICI

$h_{(n, l)}^{(t)}:$

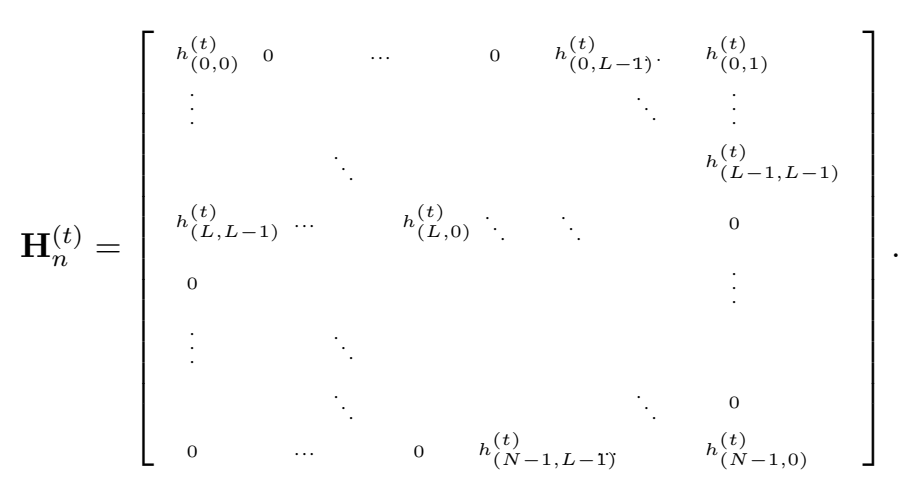

In the frequency domain, this can be written:

$$
\mathbf{Y}_{n}=\mathbf{F} \mathbf{y}_{n}=\mathbf{H}_{n} \mathbf{X}_{n}+\mathbf{U}_{n}
$$

where $\mathbf{H}_{n}=\mathbf{F} \mathbf{H}_{n}^{(t)} \mathbf{F}^{H}$ is the channel matrix, and $\mathbf{U}_{n}$ remains white Gaussian.

In case the channel is time-invariant, $h_{(n, l)}^{(t)}=h_{(m, l)}^{(t)}$ for $l=0 \ldots L-1$, and all $n, m=0 \ldots N-1$. So, in that particular case, $\mathbf{H}_{n}^{(t)}$ is circulant according to (6) and $\mathbf{H}_{n}$ is diagonal. On the contrary, the multipath aeronautical channel introduces interferences from each subcarrier to its neighbours. The channel matrix off-diagonal terms are thus not null anymore. The larger the product $f_{d T}=\Delta f_{D \max } \times T_{u}$, the heavier the consequences, where $T_{u}$ is the useful symbol duration - Table I, $\Delta f_{D \max }$ is the maximum Doppler shift relatively to the direct path Doppler shift, and $f_{d T}$ is called the normalised Doppler spread - ([26], chapter 1.2.4). So $f_{d T}$ is the ratio of the maximum Doppler shift and the intercarrier spacing. It measures the loss of orthogonality. At least, only a few neighboor subcarriers can be assumed predominant. $\mathbf{H}_{n}$ may then be banded, or taylored so as to be considered as so - [33], [39]. Fig.3 illustrates the channel matrix.

In our case, the maximum velocity of the receiver is about $v_{R X}=50 \mathrm{~m} \cdot \mathrm{s}^{-1}$. Let us consider a $600 \mathrm{MHz}$ carrier frequency, and a received path at angle $\alpha$ from the normal of the receiver. The Doppler shift relatively to the direct path can be written:

$$
\Delta f_{D}(\alpha)=\frac{v_{R X}}{\lambda}\left[\cos (\pi / 2-\alpha)-\cos \left(\pi / 2-\alpha_{D P}\right)\right]
$$

where $\alpha_{D P}$ denotes the direct path direction relatively to the receiver normal, and $\lambda$ the wavelength. If we consider 


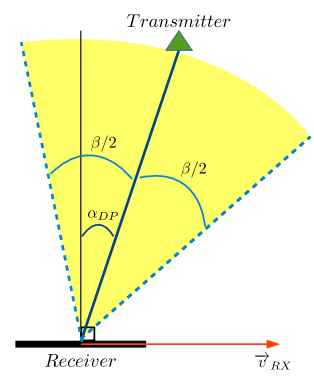

(a)

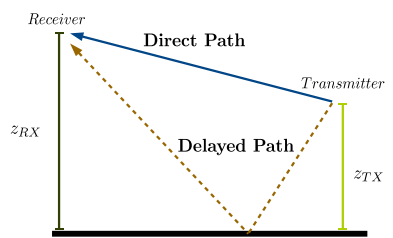

(b)

Fig. 4. Geometrical analyses to reach Doppler (a) and delay (b) estimations

that main echoes concentrate into a $\beta=60^{\circ}$ wide beam - see $4(\mathrm{a})$, the maximum Doppler shift is:

$$
\Delta f_{D \max }=\max \left[\left|\Delta f_{D}\left(\alpha_{D P}-\beta / 2\right)\right|,\left|\Delta f_{D}\left(\alpha_{D P}+\beta / 2\right)\right|\right] .
$$

Whatever $\alpha_{D P}$, it leads to: $\Delta f_{D \max } \simeq 50 \mathrm{~Hz}$. Therefore: $f_{d T} \simeq 4.5 \%$. Consequently, thanks to the slow velocity of the considered aircraft, the channel may be roughly supposed slow time varying. ICI impact may be then considered at most as limited.

\section{Reference signal misestimation impact on system detection capacity}

Before considering any detection capacity, the passive systems have to retrieve the reference signal. To quantify the impact of reference signal misestimation is in fact complex. It mainly depends on the clutter nature and on the clutter cancellation processings. However [40] highlights the detrimental influence of multipath residues in the reference signal on the cancellation capability of the algorithm proposed in [41]. Moreover [42] points that the quality of constellation estimation, that is to say of the reference signal, has a direct influence on the direct path suppression, even with different SNR.

To the same extent, we propose here to measure the benefits of a proper reference signal on detection probability thanks to a simple but representative simulation. In this simulation, we generate a DVB-T reference which is used to generate the direct path at zero delay as well as one single target delayed in time (for simplicity but without lack of representativity, no Doppler shift is considered here). We then add random errors on a few elementary symbols of the DVB-T signal in order to mimick estimation errors on the reference signal. These errors are randomly drawn in the neighbourhood of the original symbol as described in Fig.5. After direct path cancellation according to [41] using the erroneous reference signal, a Constant False Alarm Rate (CFAR) test is applied to perform the target detection, and enables to evaluate the detection probability.

Table II summarizes the parameters used for the simulations. Fig. 6 shows the detection probability versus the number of erroneous elementary symbols per OFDM symbol for different target SNR: $20 \mathrm{~dB} \leq S N R_{T G} \leq 40 \mathrm{~dB}$. These results highlight the fact that even a few number of erroneous symbols may introduce a substantial decrease on detection probability. Let us consider a $30 \mathrm{~dB}$ target SNR for instance. Only about 125 erroneous symbols over the 6817 subcarriers lead to a detection probability lower than 0.5 . The misestimation of the reference signal does not allow to properly cancel the direct path, and therefore involves higher direct path secondary lobes. Fig.7 illustrates it by providing matched filter examples for two numbers of erroneous symbols in case of $S N R_{T G}=30 \mathrm{~dB}$. The 


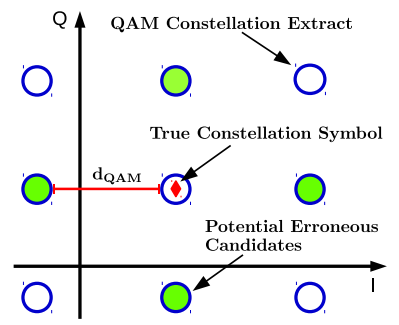

Fig. 5. Potential erroneous candidate symbols

TABLE II

SiMULATION PARAMETERS

\begin{tabular}{|c|c|}
\hline Signal & $8 \mathrm{~K}$ mode DVB-T \\
\hline Direct Path SNR & $S N R_{D P}=100 \mathrm{~dB}$ \\
\hline False alarm probability & $P_{f a}=10^{-4}$ \\
\hline Target delay & 200 samples \\
\hline Number of Monte-Carlo simulations & 2000 \\
\hline
\end{tabular}

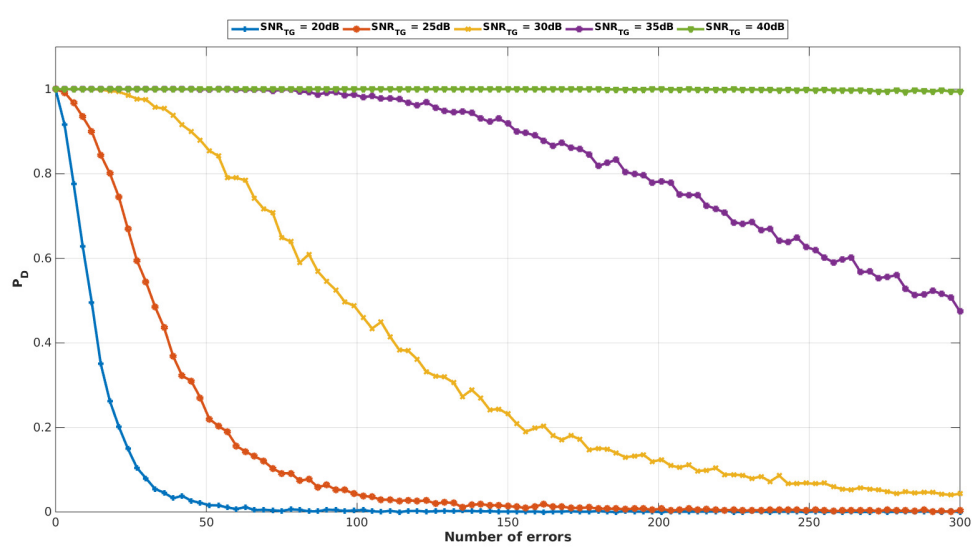

Fig. 6. Detection probability versus number of erroneous elementary symbols per OFDM symbol

secondary lobes are more than $10 \mathrm{~dB}$ stronger in case of $n_{\text {err }}=250$ erroneous symbols than in the almost error free case where $n_{e r r}=10$. As illustrated by Fig.7, the target may as a consequence be hidden under the secondary side lobe interferences.

\section{RefEREnCE Signal Estimation}

The description of the effects that the channel may have on the signal, and the impact of the reference signal misestimation on detection probability demonstrate the necessity of a relevant channel estimation method. We propose in the following a method to improve the quality of the reference signal estimate.

\section{A. Antenna Diversity}

We assume that the antenna array is composed of $N_{R X}$ sensors. Let $\mathbf{y}_{a}=\left[\mathbf{y}_{1}, \mathbf{y}_{2}, \ldots, \mathbf{y}_{N_{R X}}\right]^{T}$ denotes the received array vector. 


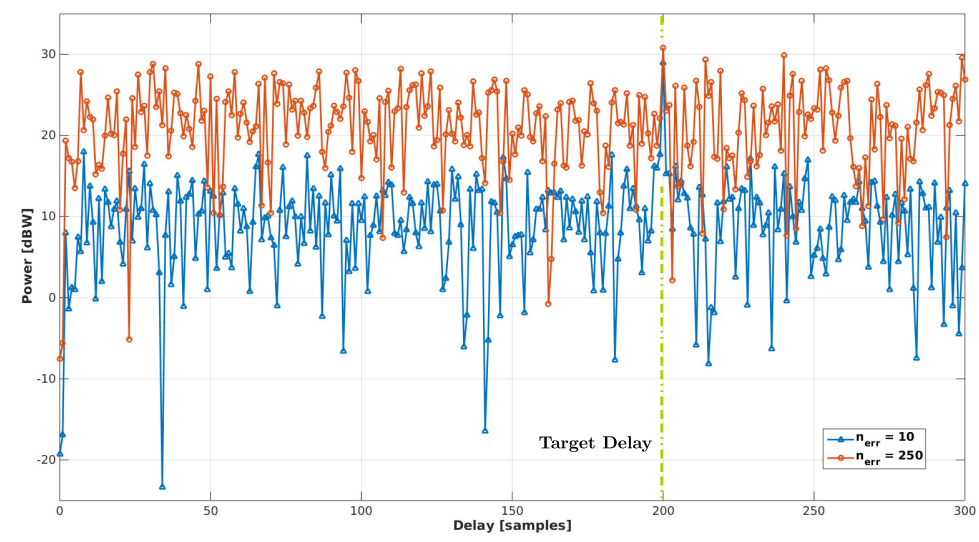

Fig. 7. Matched Filter examples after direct cancellation considering 2 different numbers of erroneous elementary symbols per OFDM symbol $-S N R_{T G}=30 \mathrm{~dB}$

Antenna diversity can help to reduce the impact of channel fluctuations due to multipath fading. The probability that each receiver channel faces simultaneously a deep fade lowers as $N_{R X}$ increases - ([43], chapter 13.4). As a consequence, antenna diversity techniques contribute to face ICI [44]. By weighting properly each array channel, these methods aim at optimizing different output parameters such as Signal-to-Noise Ratio (SNR). It is the case for Maximum Ratio Combining [44], [45]. Here we propose to focus the antenna beam in the direction provided by the maximum eigenvalue of the received signal covariance matrix $\hat{R}_{\mathbf{y}_{a} \mathbf{y}_{a}}$ :

$$
\lambda_{\max }=\max _{1, \ldots, N_{R X}} \Lambda
$$

where $\Lambda=\left[\lambda_{1}, \lambda_{2}, \ldots, \lambda_{N_{R X}}\right]$ is the eigen-value decomposition of $\hat{R}_{\mathbf{y}_{a} \mathbf{y}_{a}}$. Thus the unitary eigen-vector associated to $\lambda_{\max }$, denoted as $\boldsymbol{\nu}_{\max }$, may correspond to the direct path direction as LOS is assumed between the receiver and the transmitter. As the influence of secondary paths decreases, the proportion of useful signal increases. Therefore it also contributes to improve SNR.

The diffuse backscattered component contributions may in that way be reduced. As ICI has been previously assumed limited and, as antenna diversity helps reducing its impact, ICI is neglected in the following. The validity of this assumption was verified on the experimental data. It implies in particular that the CIR may be assumed constant during a symbol period.

From then on, y refers to the resulting received vector:

$$
\mathbf{y}=\boldsymbol{\nu}_{\max }^{T} \mathbf{y}_{a}
$$

$\mathbf{y}_{n}$ represents the $n^{\text {th }}$ OFDM symbol extracted from $\mathbf{y} . \mathbf{Y}_{n}$ denotes its frequency domain representation as expressed by (7).

\section{B. BEM Channel Model}

The aim of the BEM modeling is to represent the channel fluctuations along $N_{a}$ consecutive OFDM symbols contrary to their usual utilization to catch rapid intra-symbol variations. Let us consider a basis $\mathbf{B}$ containing $Q+1$ functions. So $Q$ corresponds to the number of functions that are used to model the channel. This parameter is 
generally set. The larger the basis, the better the channel modeling is supposed to be, but the larger the number of coefficients to estimate. Thus $Q$ is limited by the number of available equations.

The $l^{\text {th }}$ channel taps $h_{(., l)}^{(t)}$ over the $N_{a}$ symbols are described by the BEM coefficients $h_{(., l)}^{(b)}$. Its modeling by the BEM is written:

$$
h_{(n, l)}^{(t)}=\sum_{q=0}^{Q} \mathbf{B}(n, q) h_{(q, l)}^{(b)}+\epsilon_{n, l},
$$

with $\mathbf{B}$ the $N_{a} \times(Q+1)$ basis matrix, and $\epsilon_{n, l}$ the modeling error. For instance, the CE-BEM is defined as in [34]:

$$
\mathbf{B}(n, q)=\exp \left(j \frac{2 \pi n}{N}\left(q-\frac{Q}{2}\right)\right) .
$$

We introduce $\mathbf{h}_{q}=\left[h_{(q, 0)}^{(b)}, \ldots, h_{(q, L-1)}^{(b)}\right]^{T}$ the decomposition coefficients for the $q^{\text {th }}$ function, $q=0 \ldots Q$. So it comes that:

$$
\mathbf{H}_{n}^{(t)}=\sum_{q=0}^{Q} \mathbf{B}(n, q) \mathbf{H}^{(q)},
$$

where $\mathbf{H}^{(q)}$ is the circulant matrix defined from $\mathbf{h}_{q}$ :

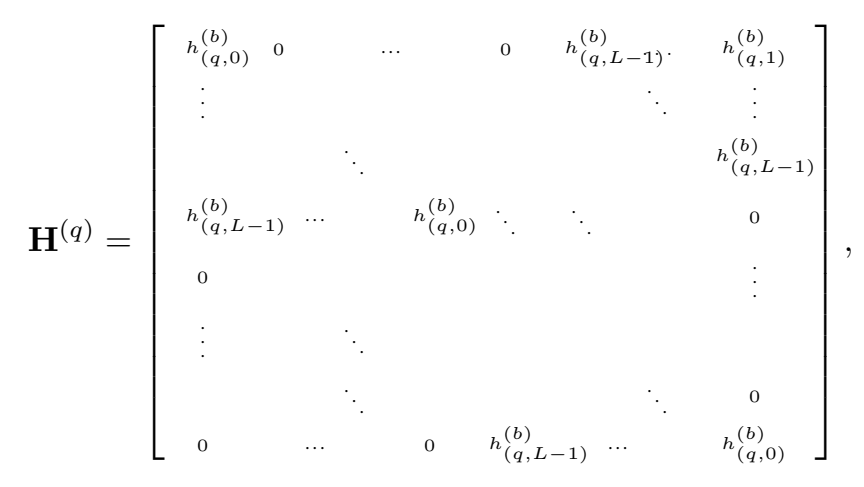

Therefore :

$$
\mathbf{H}_{n}=\sum_{q=0}^{Q} \mathbf{B}(n, q) \mathbf{F} \mathbf{H}^{(q)} \mathbf{F}^{H} .
$$

Let us denote: $\Delta_{q}=\mathbf{F} \mathbf{H}^{(q)} \mathbf{F}^{H}$. As used in [34]: $\Delta_{q}=\operatorname{diag}\left(\sqrt{N} \mathbf{F}_{L} \mathbf{h}_{q}\right)$, where $\mathbf{F}_{L}$ is the restriction of $\mathbf{F}$ to the $L$ first columns. Finally:

$$
\mathbf{Y}_{n}=\sum_{q=0}^{Q} \mathbf{B}(n, q) \Delta_{q} \mathbf{X}_{n}+\mathbf{V}_{n}
$$

$\mathbf{V}_{n}$ expresses at once the channel additive noise and the modeling error. The $L$ pilot rows of the previous equation can be extracted, so that:

$$
\mathbf{Y}_{n}^{(p)}=\sum_{q=0}^{Q} \mathbf{B}(n, q) \Delta_{q}^{(p)} \mathbf{X}_{n}^{(p)}+\mathbf{V}_{n}^{(p)}
$$

From the definition of $\Delta_{q}$, it can be further shown that $\Delta_{q}^{(p)}$ can be inferred from the $L$-sized Fourier transform of $\mathbf{h}_{q}$.

The ratio $\mathbf{Y}_{n}^{(p)}(m) / \mathbf{X}_{n}^{(p)}(m)$ gives a sampled estimation of the CFR at the $m^{\text {th }}$ pilot. Classic demodulation uses them to compute the whole CFR by interpolation, independently over each OFDM symbol. 
Here it comes:

$$
\frac{\mathbf{Y}_{n}^{(p)}(m)}{\mathbf{X}_{n}^{(p)}(m)}=\left[\mathbf{B}_{(n, 0)} \ldots \mathbf{B}_{(n, Q)}\right]\left[\begin{array}{c}
\Delta_{0}^{(p)}(m, m) \\
\vdots \\
\Delta_{Q}^{(p)}(m, m)
\end{array}\right]+\mathbf{V}_{n}^{(p)}(m)
$$

As a consequence:

$$
\left[\begin{array}{c}
\frac{\mathbf{Y}_{0}^{(p)}(m)}{\mathbf{X}_{0}^{(p)}(m)} \\
\vdots \\
\frac{\mathbf{Y}_{N a-1}^{(p)}(m)}{\mathbf{X}_{N_{a}-1}^{(p)}(m)}
\end{array}\right]=\mathbf{B}\left[\begin{array}{c}
\Delta_{0}^{(p)}(m, m) \\
\vdots \\
\Delta_{Q}^{(p)}(m, m)
\end{array}\right]+\left[\begin{array}{c}
\mathbf{V}_{0}^{(p)}(m) \\
\vdots \\
\mathbf{V}_{N_{a}-1}^{(p)}(m)
\end{array}\right]
$$

Finally:

$$
\mathbf{T}=\mathbf{B} \boldsymbol{\Delta}^{(p)}+\mathbf{V}
$$

where $\mathbf{T}$ is the matrix whose columns are composed of the ratio of the received sample over the transmitted pilot, for one pilot over $N_{a}$ symbols, and $\boldsymbol{\Delta}^{(p)}$ is the matrix whose the $m^{\text {th }}$ column is composed of the $m^{\text {th }}$ diagonal value of $\Delta_{q}^{(p)}$ for $q=0 \ldots Q$. Equation (21) allows to estimate $\boldsymbol{\Delta}^{(p)}$ from $\mathbf{T}$, and therefore to get the BEM coefficients.

The channel matrix is then inferred. As ICI has been assumed negligible, $\mathbf{H}_{n}$ may be considered as diagonal, for off-diagonal terms may be neglected. As a consequence, equalization is made simple. The estimated OFDM symbol is derived from the ratio of the received vector over the estimated CFR. The equalization process provides for the $k^{t h}$ subcarrier a complex value $\tilde{\mathbf{X}}_{n}(k)$ corresponding to the in-phase and quadrature components. This estimated point is then mapped to the exact transmitted constellation point $\hat{\mathbf{X}}_{n}(k)$.

\section{Previous Model Adaptation}

At signal reception, time synchronization provides the sample index of the beginning of one OFDM symbol. However it only detects the main path. But the DVB-T networks may be a Single Frequency Network (SFN), implying that it may exist a re-emitter, closer than the main broadcaster from the receiver. Besides artificial delays may be used for the purpose of network optimization. In any case, it is highly unlikely that the main path delay falls exactly on the sample grid. Thus sidelobes are generated, which spread apart from the main path sample. As a consequence, we use a delay guard. It means that we voluntarily set the symbol beginning a few samples before. Let $\tau_{G}$ denotes this sampled delay.

In other words, we aim at estimating the channel response $h_{\left(n, l-\tau_{G}\right)}^{(t)}$ for the $n^{t h}$ symbol. So, according to Fourier transform properties, this $\tau_{G}$ time translation equates to a frequency domain modulation. At the $m^{\text {th }}$ subcarrier, it expresses as:

$$
\Phi(m)=2 \pi \frac{m \tau_{G}}{N} .
$$

It is especially true at pilot index. However in the DVB-T case, the pilots positions are shifted by 3 subcarriers from one symbol to the next, as previously detailed - see Fig.1. So the experienced modulation at the $m^{\text {th }}$ pilot of the $n^{t h}$ symbol can be precised - see (1):

$$
\Phi\left(P_{n}(m)\right)=2 \pi\left(\frac{m D_{f} \tau_{G}}{N}+\frac{d_{n} \tau_{G}}{N}\right) .
$$




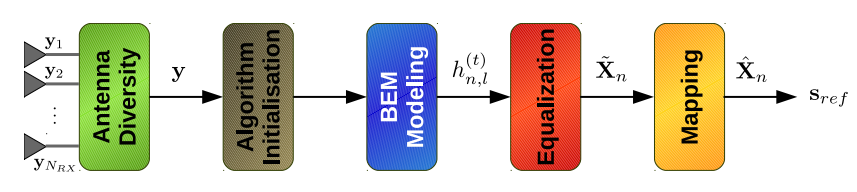

Fig. 8. Algorithm overview

So the combination of delay guard and pilot index shift, provokes a phase shift which is composed of a modulated and a constant part. This has no consequence if only one symbol is considered. This is for instance the case of terrestrial stationary systems using the classic OFDM demodulation. Indeed the translated CFR samples at pilot index, helps estimating the $\tau_{G}$-delayed CIR, as (23) expresses.

Yet in the present case, several consecutive symbols are used to catch channel time variations. Consequently from the $n^{t h}$ symbol to the first, it exists a constant phase shift component $\Delta \Phi_{n}=\Phi\left(P_{n}(m)\right)-\Phi\left(P_{0}(m)\right)$ for all $m=0 \ldots L-1$ :

$$
\begin{aligned}
& \Delta \Phi_{n}=2 \pi\left(\frac{d_{n} \tau_{G}}{N}-\frac{d_{0} \tau_{G}}{N}\right), \\
& \Delta \Phi_{n}=2 \pi \frac{d_{n} \tau_{G}}{N} .
\end{aligned}
$$

as $d_{0}=0$, as stated in section II-A. This constant phase shift translates into a rotation of the estimated QAMconstellation.

Two solutions can be considered to cope with this undesired effect. The first solution consists in creating a regular pilot-grid by interpolation of the pilots every 4 subcarriers for every OFDM symbol. The second one imposes to transpose the pilot-based CFR into the time domain, in order to compensate the time modulation due to the pilot location shift and then to get back to the frequency domain. Here we resort to the first solution and initialize the BEM algorithm with a regular pilot pattern both in frequency and time dimension, derived from interpolation.

Fig. 8 provides an overview of the proposed algorithm.

\section{EXPERIMENTAL RESULTS}

\section{A. Experimental Flight Tests}

A flight test took place in South East of France. Data were recorded from the Mont Ventoux broadcaster:

- Effective Radiated Power (ERP): $50 \mathrm{~kW}$

- Transmitter altitude: $1900 \mathrm{~m}$

- Coarse transmitter to receiver distance: $47 \mathrm{~km}$

The DVB-T channels are transmitted over carrier frequencies from $570 \mathrm{MHz}$ to $618 \mathrm{MHz}$. The DVB-T mode $8 \mathrm{~K}$ is used, as previously described in II-A. A dedicated antenna, named Rivera, has been designed for the project. The main constraint comes from the necessity to fit the antenna array in the space available in the pod of the Busard motorglider (around $110 \mathrm{~cm}$ available) while the wavelength in the DVB-T dedicated frequency band may be up to $60 \mathrm{~cm}$ - Fig.9. The $N_{R X}=3$ receiving channels are disposed in a side-looking configuration. During the experimental flight, the aircraft speed was comprised bewteen 45 and $50 \mathrm{~m} \cdot \mathrm{s}^{-1}$, and the altitude between 1000 and $1500 \mathrm{~m}$ above ground level. 


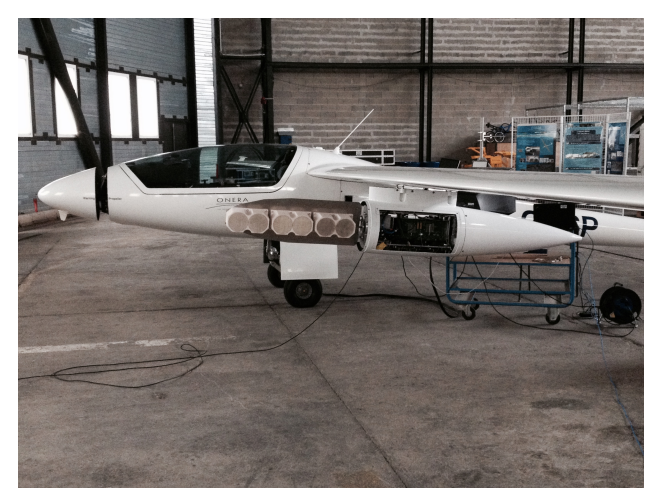

Fig. 9. Busard Onera motorglider and Rivera antenna

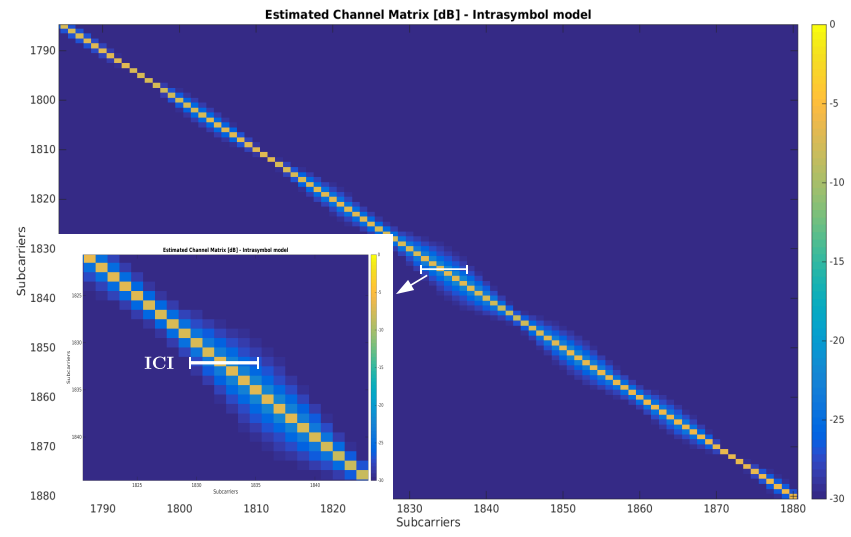

Fig. 10. Estimated channel matrix - intrasymbol time variation model

\section{B. Channel Estimation}

To analyse the potential ICI, we use the linear intra-symbol model of the channel time variations as proposed by [24] using the signal received on one of the three channels. Fig.10 shows an extraction of the corresponding estimated channel matrix. The zoom view highlights that ICI spreads apart from the diagonal over a few subcarriers only. The channel matrix decrease from the diagonal to the outside reaches up to $30 \mathrm{~dB}$ over these maximum five subcarriers. It confirms our assumption that ICI may be neglected.

The BEM-based method has been built with $N_{a}=32$ consecutive symbols. The basis $\mathbf{B}$ is provided by the exponential BEM, as defined by (13), as it aims at modeling Doppler-induced CIR phase evolution. $Q$ has been empirically set to 32 .

Fig. 11 displays the CFR for 3 different symbols. There are 8 OFDM symbols in between each of the considered symbols. It highlights the deep fades that characterise the channel we are facing. It can also be noticed that these fades are evolving along time. So the channel estimation has to cope with fading effects and to model their time fluctuations. 


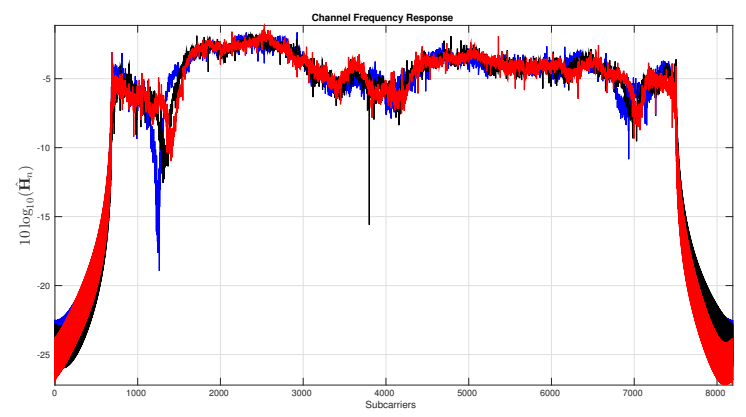

Fig. 11. Estimated channel frequency response for three OFDM different symbols - every 8 symbols

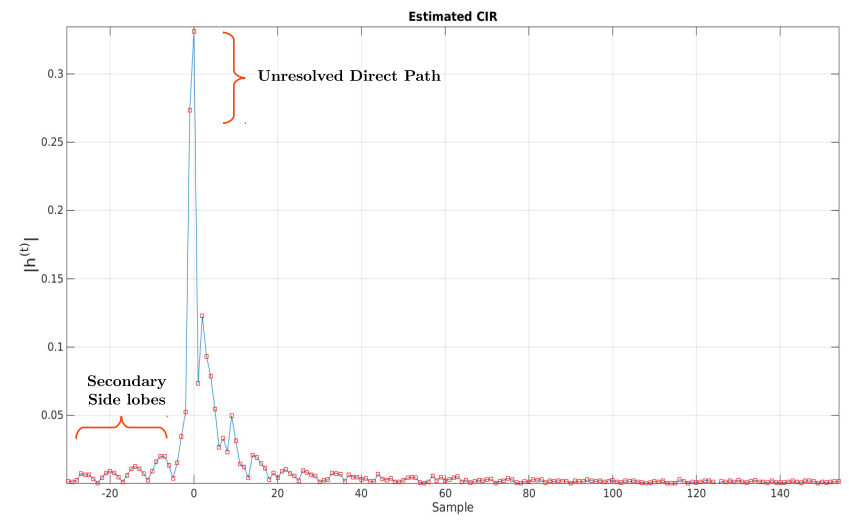

Fig. 12. Estimated channel impulse response - delay guard necessity

\section{Model Initialization Necessity}

Fig.12 displays the estimated CIR for one symbol only. The direct path is obvious. It appears that it is unresolved. The fact that it falls between two samples, induces secondary sides lobes apart from the main taps toward the negative delay domain in particular. Even if the present case does not correspond to a SFN environment, Fig.12 justifies the necessity to introduce a delay guard.

Such a delay guard induces a rotation of the constellation according to III-C if no previous initialization of the BEM model is performed. The rotation angle depends on the symbol type, that is the symbol number modulo 4. As expressed by (24), in the present case, a delay guard of 30 samples implies a rotation angle that is a multiple of $3.95^{\circ}$. Fig. 13 shows the rotated constellations, for each symbol type. The angle may be estimated from the slope of the straight line joining the pilots. And Fig.14 points the estimated angles for 32 consecutive symbols. It highlights the periodicity of their value, and confirms the theoretical analysis.

\section{Reference Signal Estimation}

To quantify the performance of the different algorithms, we use the Modulation Error Rate (MER) ([46], chapter 9.18.2). MER corresponds to the ratio of the sum of the power of the ideal constellation symbol vectors over the 

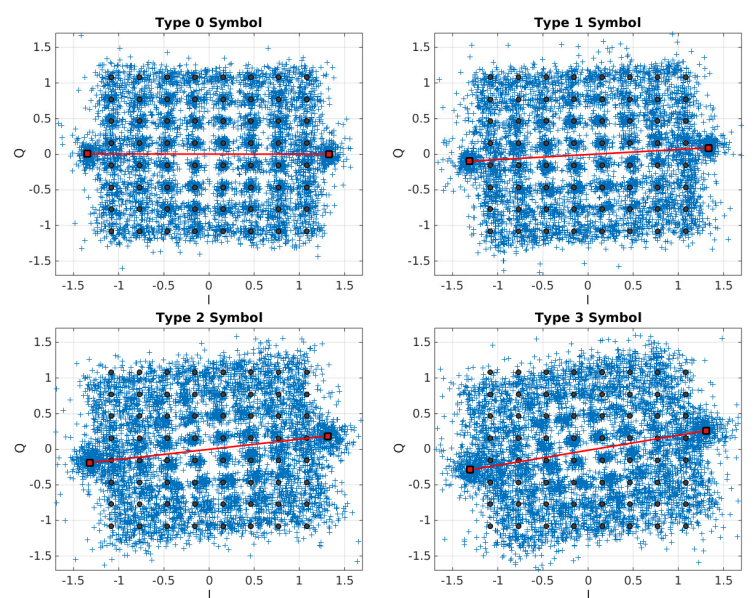

Fig. 13. Constellation rotation $-\tau_{G}=30$

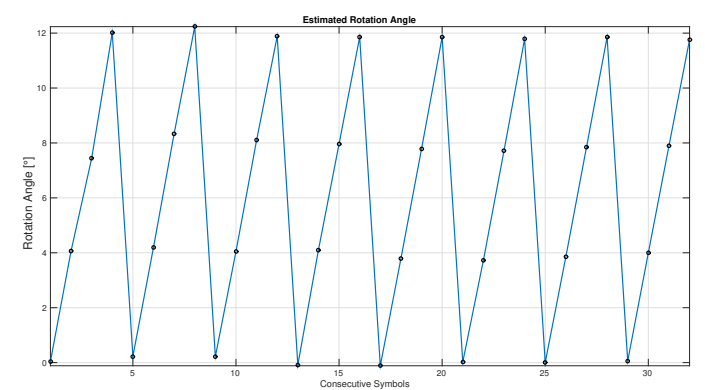

Fig. 14. Estimated rotation angle for 32 consecutive symbols

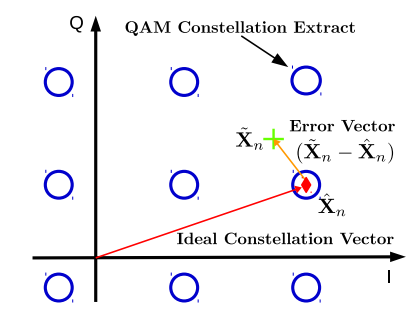

Fig. 15. Schematic definition of MER

sum of the power of error vectors, as illustrated by 15, and defined by:

$$
M E R=10 \times \log _{10}\left(\frac{\sum_{n, k}\left|\hat{\mathbf{X}}_{n}(k)\right|^{2}}{\sum_{n, k}\left|\tilde{\mathbf{X}}_{n}(k)-\hat{\mathbf{X}}_{n}(k)\right|^{2}}\right)
$$

where $\tilde{\mathbf{X}}_{n}(k)$ represents the estimated point and $\hat{\mathbf{X}}_{n}(k)$ the closest constellation point for each subcarrier $k^{t h}$. MER can be seen as a form of SNR that gives an indication of the estimation relevancy.

Fig.16 presents the performance obtained for different demodulation methods. We compare our method to four different algorithms and use the classic demodulation as a reference, where the CFR is inferred from interpolation of its pilot-based LS estimate. We first evaluate the performances of the intra-symbol linear model proposed in [24], and an intra-symbol BEM algorithm with a polynomial basis and $Q=2$ [34], [35]. It clearly points the fact 


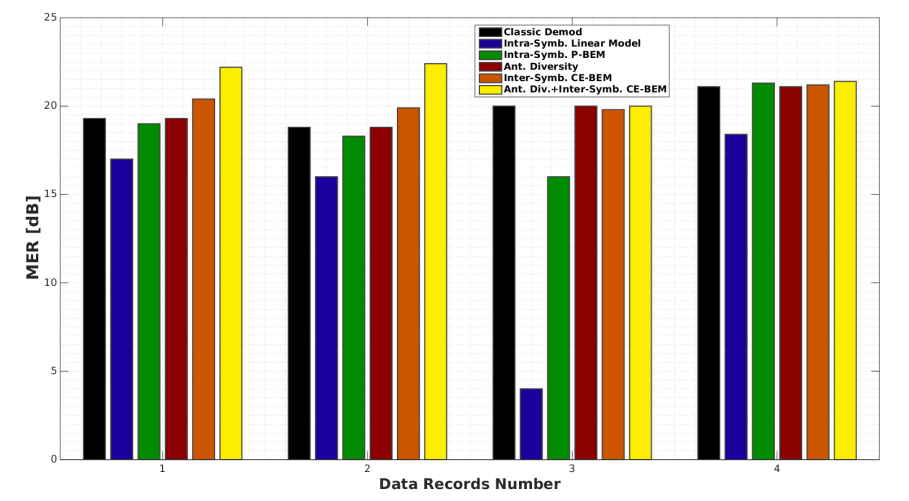

Fig. 16. Measured MER for 4 data records - classic demodulation (black), intra-symbol linear model (blue), intra-symbol BEM (green), antenna diversity (red), BEM inter-symbol (orange), antenna diversity and inter-symbol BEM (yellow)

that intra-symbol channel variations modeling does not fit the considered aeronautical channel. The attempt to estimate ICI may introduce estimation disturbances. It emphasizes the benefits of inter-symbol model.

Then two other methods are compared: the classic demodulation that uses the antenna diversity combined signal, as explained in section III-A, and the BEM only inter-symbol model. A general trend can be derived from these examples. The antenna diversity and the BEM modeling provide each a substantial improvement. The first one underlines the benefits of antenna diversity against the diffuse backscattered components, whereas the second one points the positive impact of the inter-symbol model. However our method, by combining both, enables even better performance. The results are all the more interesting that the estimation is more homogeneous over the QAM-constellation than in the classic case where the edges undergo a heavier channel impact as it can be seen in Fig.17 that shows the estimated constellations obtained with the four methods for the data record number 2 . Three OFDM symbols are superimposed for each subfigure.

For data record 3, classic demodulation performs equivalently to our method. It may be explained by the fact that some of the demodulated points, $\tilde{\mathbf{X}}_{n}(k)$, lie farther from the edge of constellation with our method than with the classic demodulation. Refering to the MER definition (25), this may impair the MER denominator and decrease the global MER. However the overall benefits of our method is clearly visible on Fig.17.

The MER can also be estimated for the simulated case of section II-C. The power of the ideal constellation vectors may be average by the average power of equiprobable QAM symbols, and the power of the error vectors may be approximated by : $n_{e r r} \times d_{Q A M}$, where $n_{e r r}$ is the number of errors and $d_{Q A M}$ corresponds to the distance between two constellation points as shown in Fig.5. Fig.18 displays the resulting MER. Only a few dB MER improvement may involve a large decrease of the number of erroneous symbols. Let us consider data record 2: with our method, $M E R=22.4 \mathrm{~dB}$, whereas $M E R=18.8 \mathrm{~dB}$ with the classic demodulation. It respectively corresponds to the following numbers of erroneous symbols: $n_{\text {err }}=110$ and $n_{\text {err }}=260$. These reference signal improvements may be translated into detection probability improvements in accordance with Fig. 6 in the simple simulated case of section II-C. In case of $S N R_{T G}=30 \mathrm{~dB}$, it means $P_{D}>0.5$ with our method, but $P_{D}<0.1$ with the classic demodulation. So the reference signal method we propose, may have worthwhile impact on the detection capacity of the system. 


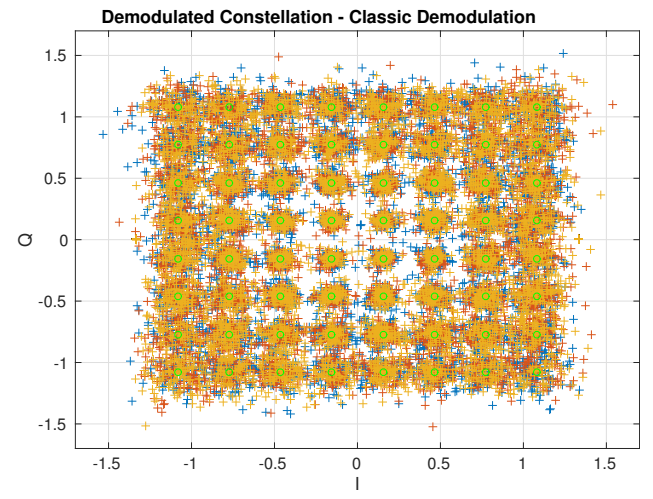

(a) Classic Demodulation

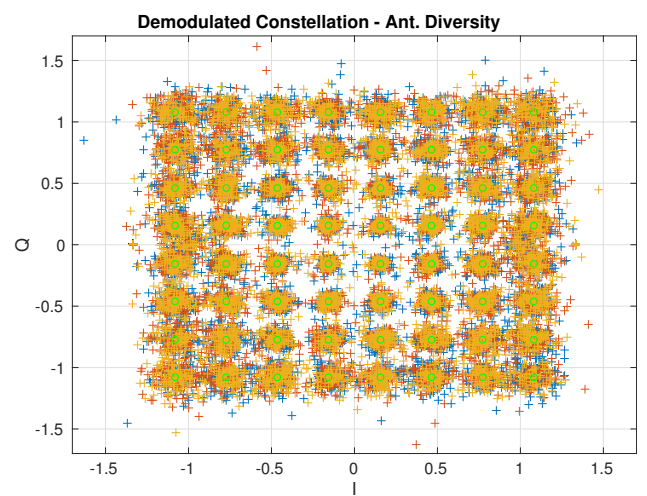

(c) Antenna Diversity

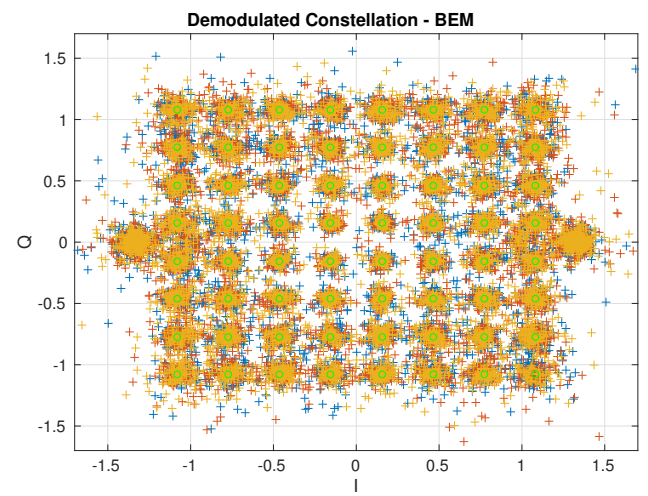

(b) BEM

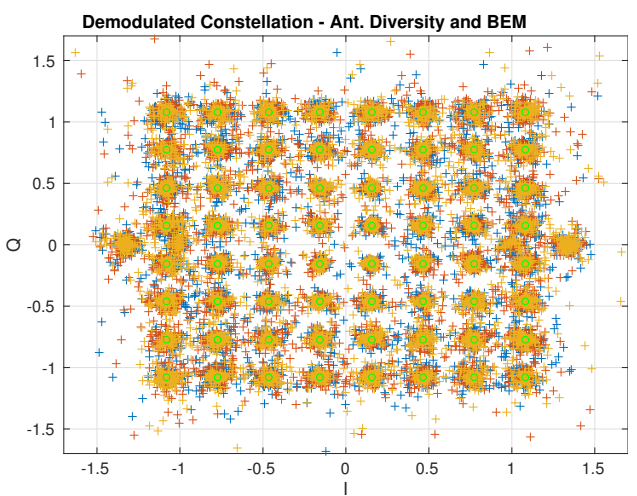

(d) Antenna Diversity + BEM

Fig. 17. Data record 2 - demodulated constellation with 4 different methods

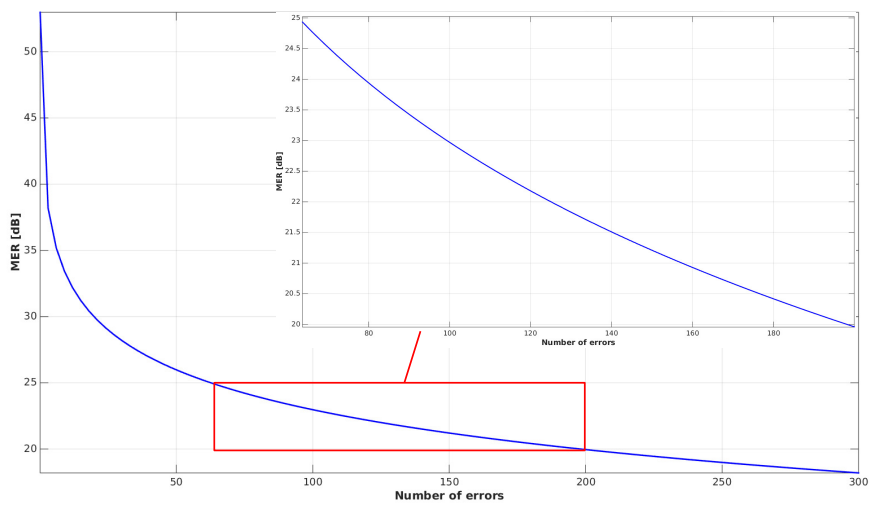

Fig. 18. MER versus number of erroneous symbols per OFDM symbol

\section{CONClusion}

Passive airborne radar is a recent topic of research. Theoretical studies have established its feasibility, and first experiments have confirmed it. Even if stationary bistatic system have produced a substantial literature, adapted signal processing need to be developped to ensure detection from an airborne plateform. Especially we have pointed out the harsh impact of the aeronautical channel on the DVB-T OFDM system. The composite nature of the received signal makes it difficult to extract the direct path component. Moreover the mobility of the receiver 
implies channel time fluctuations, that may cause ICI. Nevertheless the low velocity of the considered plateform, and the use of antenna diversity allow us to neglect this complex effect. Despite this, a suitable method had to be developped to catch channel inter-symbol variations. Thus we derived a BEM-based solution that enables us to model the channel tap variations over several consecutive symbols. The experimental results obtained by the application of the proposed method to real data measurements confirm the efficiency of combining antenna diversity and BEM to face the aeronautical channel induced disruptions.

Thanks to our method, we went through the first step of passive detection, that consists in retrieving a proper reference signal. Yet we have now to proceed forward signal processing in order to cancel the direct path and suppress clutter echoes. Then it will be possible to quantify the influence of a well-estimated reference on these algorithms. This will be the object of a subsequent paper.

\section{ACKNOWLEDGMENT}

The authors would like to thank Fabrice Boust (SONDRA-ONERA), Jean-François Nouvel (ONERA) and Serge ROQUES (ONERA) for their help in the antenna design and the conduct of flight trials. Besides they would like to thank TDF for providing useful information about DVB-T broadcasting,

\section{REFERENCES}

[1] F. Colone, D. O'Hagan, P. Lombardo, and C. Baker, "A multistage processing algorithm for disturbance removal and target detection in passive bistatic radar," IEEE Transactions on Aerospace and Electronic Systems, vol. 45, no. 2, pp. 698-722, 2009.

[2] C. R. Berger, B. Demissie, J. Heckenbach, P. Willett, and S. Zhou, "Signal processing for passive radar using OFDM waveforms," J. Sel. Topics Signal Processing, vol. 4, pp. 226-238, 2010.

[3] D. Poullin, "Passive detection using digital broadcasters (DAB, DVB) with COFDM," IEEE Proceedings Radar, Sonar and Navigation, vol. 152, no. 3, pp. 143-152, 2005.

[4] J. Raout, A. Santori, and E. Moreau, "Passive bistatic noise radar using DVB-T signals," IET Radar, Sonar and Navigation, vol. 4, no. 3, pp. 403-411, 2010.

[5] J. E. Palmer, H. A. Harms, S. J. Searle, and L. M. Davis, "DVB-T passive radar signal processing," IEEE Trans. Signal Processing, vol. 61, pp. 2116-2126, 2013.

[6] P. Falcone, F. Colone, and P. Lombardo, "Potentialities and challenges of WiFi-based passive radar," IEEE Aerospace and Electronic Systems Magazine, vol. 27, no. 11, pp. 15-26, 2012.

[7] A. Schroeder and M. Edrich, "CASSIDIAN multiband mobile passive radar system," Radar Symposium IRS, Proceedings International, 2011.

[8] J. Raout, "Sea target detection using passive DVB-T based radar," International Conference on Radar, pp. 695-700, 2008.

[9] D. O’Hagan, A. Capria, D. Petri, V. Kubica, M. Greco, F. Berizzi, and A. Stove, "Passive bistatic radar (PBR) for harbour protection applications," in 2012 IEEE Radar Conference. IEEE, 2012, pp. 0446-0450.

[10] K. Kulpa, M. Malanowski, P. Samczynski, and B. Dawidowicz, "The concept of airborne passive radar," Microwaves, Radar and Remote Sensing Symposium (MRRS), pp. 267-270, 2011.

[11] D. K. P. Tan, M. Lesturgie, H. Sun, and Y. Lu, "Target detection performance analysis for airborne passive bistatic radar," in IGARSS, 2010, pp. 3553-3556.

[12] D. Tan, M. Lesturgie, H. Sun, and Y. Lu, "Signal analysis of airborne passive radar using transmissions of opportunity," IEEE CIE International Conference on Radar, pp. 169-172, 2011.

[13] J. Brown, K. Woodbridge, A. Stove, and S. Watts, "VHF airborne passive bistatic radar ground clutter investigation," IET Radar Conference, vol. 27, no. 11, pp. 53-53(1), 2012.

[14] J. Brown, K. Woodbridge, and H. Griffiths, "Passive radar experiments from an airborn plateform," IEEE Aerospace and Electronic Systems Magazine, vol. 27, no. 11, pp. 50-55, 2012.

[15] B. Dawidowicz, K. Kulpa, M. Malanowski, J. Misiurewicz, P. Samczynski, and M. Smolarczyk, "DVB-T passive radar signal processing," IEEE Aerospace and Electronic Systems Magazine, vol. 48, no. 2, pp. 1347-1357, 2012. 
[16] B. Dawidowicz, P. Samczynski, M. Malanowski, J. Misiurewicz, and K. Kulpa, "Detection of moving target with multichannel airborne passive radar," IEEE Aerospace and Electronic Systems Magazine, vol. 27, no. 11, pp. 42-49, 2012.

[17] L. Ulander et al., "Bistatic VHF/UHF-band airborne SAR experiment," IET Conference Proceedings, pp. 72-72(1), 2012.

[18] L. Ulander, P.-O. Frölind, A. Gustavsson, R. Ragnarsson, and G. Stenström, "VHF/UHF bistatic and passive SAR ground imaging," IEEE Radar Conference, pp. 669-673, 2015.

[19] Digital Video Broadcasting (DVB); Framing structure, channel coding and modulation for digital terrestrial television, ETSI Std. ETSI EN 300744 V1.6.1, 2009.

[20] E. Haas, "Aeronautical channel modeling," Vehicular Technology, IEEE Transactions on, vol. 51, no. 2, pp. 254-264, Mar 2002.

[21] P. Bello, "Aeronautical channel characterization," Communications, IEEE Transactions on, vol. 21, no. 5, pp. 548-563, May 1973.

[22] P. Hoeher, S. Kaiser, and P. Robertson, "Two-dimensional pilot-symbol-aided channel estimation by Wiener filtering," in IEEE ICASSP, 1997, pp. 1845-1848.

[23] S. Tomasin and M. Butussi, "Analysis of interpolated channel estimation for mobile OFDM systems," IEEE Trans. Communications, vol. 58, pp. 1578-1588, 2010.

[24] Y. Mostofi and D. C. Cox, "Ici mitigation for pilot-aided OFDM mobile systems," Trans. Wireless. Comm., vol. 4, no. 2, pp. 765-774, Mar. 2005.

[25] H. Hijazi and L. Ros, "Polynomial estimation of time-varying multipath gains with intercarrier interference mitigation in OFDM systems," IEEE Trans. Vehicular Technology, vol. 58, pp. 140-151, 2009.

[26] H. Hijazi, "High speed radio-mobile channel estimation in OFDM systems," Theses, Institut National Polytechnique de Grenoble - INPG, Nov. 2008, équipe C2S du Département Images et Signal (DIS).

[27] B. Yang, K. B. Letaief, R. S. Cheng, and Z. Cao, "Channel estimation for OFDM transmission in multipath fading channels based on parametric channel modeling," IEEE Trans. Communications, vol. 49, pp. 467-479, 2001.

[28] C. Berthillot, A. Santori, O. Rabaste, D. Poullin, and M. Lesturgie, "Improving bem channel estimation for airborne passive radar reference signal reconstruction," in The International Radar Symposium 2015 (IRS 2015), Dresden, Germany, Jun. 2015, pp. pp 77-82.

[29] M.Poggioni, L. Rugini, and P. Banelli, "Multistage decoding-aided channel estimation and equalization for DVB-H in single-frequency networks," IEEE SPAWC 09, pp. 181-185, 2009.

[30] H. Hijazi and L. Ros, "Joint data QR-detection and kalman estimation for OFDM time-varying Rayleigh channel complex gains," IEEE Transactions on Communications, vol. 58, no. 1, pp. 170-178, Jan. 2010.

[31] G. B. Giannakis and C. Tepedelenlioglu, "Basis expansion models and diversity techniques for blind identification and equalization of time-varying channels," IEEE Proc., vol. 86, pp. 1969-1986, 1998.

[32] I. Barhumi, G. Leus, and M. Moonen, "Time-varying fir equalization of doubly selective channels," IEEE Trans. Wireless Commu., vol. 4, pp. 202-214, 2005.

[33] L. Rugini, P. Banelli, and G. Leus, "Low-complexity banded equalizers for OFDM systems in doppler spread channels," EURASIP J. Adv. Sig. Proc., vol. 2006, 2006.

[34] Z. Tang, R. C. Cannizzaro, G. Leus, and P. Banelli, "Pilot-assisted time-varying channel estimation for OFDM systems," IEEE Trans. Signal Processing, vol. 55, pp. 2226-2238, 2007.

[35] G. Tauböck, F. Hlawatsch, D. Eiwen, and H. Rauhut, "Compressive estimation of doubly selective channels in multicarrier systems: Leakage effects and sparsity-enhancing processing,” J. Sel. Topics Signal Processing, vol. 4, pp. 255-271, 2010.

[36] M. Visitin, "Karhunen-loeve expansion of a fast rayleigh fading process," Electronics Letters, vol. 32, no. 18, 1996.

[37] T. Zemen and C. F. Mecklenbräuker, "Time-variant channel estimation using discrete prolate spheroidal sequences," IEEE Trans. Signal Processing, vol. 53, pp. 3597-3607, 2005.

[38] Z. Tang, G. Leus, R. C. Cannizzaro, and P. Banelli, "Pilot-assisted time-varying OFDM channel estimation based on multiple OFDM symbols," in in Proc. Workshop on Signal Processing Advances in Wireless Communications (SPAWC), 2006.

[39] P. Schniter, "Low-complexity equalization of OFDM in doubly selective channels," IEEE Trans. Signal Processing, vol. 52, pp. 1002-1011, 2004.

[40] R. Cardinali, F. Colone, P. Lombardo, O. Crognale, A. Cosmi, and A. Lauri, "Multipath cancellation on reference antenna for passive radar which exploits FM transmission," IET International Conference on Radar Systems, 2007.

[41] F. Colone, R. Cardinali, and P. Lombardo, "cancellation clutter and multipath in passive radar using a sequential approach," IEEE 2006 Radar Conference, vol. 45, pp. 393-399, 2006.

[42] H. Tang, X. Wan, L. Hong, W. Chen, and J. Yi, "Detection improvement by modified modulation error rate of reference signal in passive radar," General Assembly and Scientific Symposium (URSI GASS), 2014. 
[43] J. Proakis and M. Salehi, Digital Communications, 5th Edition, 5th ed. McGraw-Hill Science/Engineering/Math, 112007.

[44] A. Hutter, J. S. Hammerschmidt, E. de Carvalho, and J. M. Cioffi, "Receive diversity for mobile OFDM systems," in WCNC, 2000.

[45] R. H. A. Salem, B. Eitel, and J. Speidel, "Enhanced maximum ratio combining for mobile DVB-T reception in doubly selective channels," in VTC Spring, 2015.

[46] Digital Video Broadcasting (DVB); Measurement guidelines for DVB systems, ETSI Std. ETSI TR 101 290 V1.2.1, 2001. 